\title{
Separability of rank two quantum states on multiple quantum spaces
}

\author{
Shao-Ming Fei ${ }^{1}{ }^{2}$, Xiu-Hong Gao ${ }^{1}$, Xiao-Hong Wang ${ }^{1}$, Zhi-Xi Wang ${ }^{1}$, and Ke $\mathrm{Wu}^{1}$ \\ ${ }^{1}$ Department of Mathematics, Capital Normal University, Beijing, China. \\ ${ }^{2}$ Institute of Applied Mathematics, University of Bonn, 53115 Bonn Germany
}

\begin{abstract}
Explicit sufficient and necessary conditions for separability of $N$-dimensional rank two multiparty quantum mixed states are presented. A nonseparability inequality is also given, for the case where one of the eigenvectors corresponding to nonzero eigenvalues of the density matrix is maximally entangled.
\end{abstract}

PACS: 03.65.Bz;89.70.+c

Quantum entanglement is playing an essential role in quantum information processing (cf. [1, 2, 3]). The separability of pure states for bipartite systems is quite well understood (cf. (4). For mixed states, some progress has been achieved in understanding the separability and entanglement problem for bipartite systems (cf. [5]), e.g., the proper definition of separable and entangled states formulated by Werner [6], the Peres [7] criterion that all separable states necessarily have a positive partial transpose, which is further shown to be also a sufficient condition for separability in $2 \times 2$ and $2 \times 3$ systems [8, 9].

The multiparty entangled states have been investigated recently [10, 11]. These states are also of importance in quantum information processing, for instance, three party entanglement of the GHZ type can allow for interesting applications such as quantum secret sharing [12], and many experimental groups have tried to generate such states [13. However less is known about how to characterize multiparty entanglements completely. The maximum connectedness, persistency [14 and Schmidt measure 15] have been used in partly describing entanglements of pure multiparty states. There exists no general criterion that allows one to distinguish whether a general mixed state is separable or not. In [16] the separability and entanglement in $2 \times 2 \times N$ composite quantum systems have been studied.

In this paper, we study sufficient and necessary conditions for separability of higher-dimensional quantum systems on $H \otimes H \otimes \ldots \otimes H$, which generalize the results in [17]. In particular, we consider density matrices with rank two. The separability condition for these kind of mixed states in arbitrary dimensions is explicitly given. In addition, we present a nonseparability inequality valid in the case where one of the eigenvectors corresponding to nonzero eigenvalues of a density matrix is maximally entangled.

We first consider the case of three $N$-dimensional quantum spaces. Let $H$ be an $N$ dimensional complex Hilbert space, with $e_{i}, i=1,2, \ldots, N$, an orthonormal basis. A general 
pure state on $H \otimes H \otimes H$ is of the form

$$
|\Psi\rangle=\sum_{i, j, k=1}^{N} a_{i j k} e_{i} \otimes e_{j} \otimes e_{k}, \quad a_{i j k} \in \mathbb{C}
$$

with the normalization $\sum_{i, j, k=1}^{N} a_{i j k} a_{i j k}^{*}=1$ (* denoting complex conjugation).

Let $U$ denote a unitary transformation on the Hilbert space $H$, such that

$$
U e_{i}=\sum_{j=1}^{N} b_{i j} e_{j}, \quad b_{i j} \in \mathbb{C}
$$

and $\sum_{j=1}^{N} b_{i j} b_{k j}^{*}=\delta_{i k}$ (with $\delta_{i k}$ the usual Kronecker's symbol ). We call a quantity an invariant associated with the state $|\Psi\rangle$ if it is invariant under all local unitary transformations of $U_{1} \otimes U_{2} \otimes$ $U_{3}$. By generalizing the results of analysis on invariants for qubits [18], the following quantities are invariants [19] under local unitary transformations:

$$
\begin{aligned}
I_{0} & =\sum_{i, j, k=1}^{N} a_{i j k} a_{i j k}^{*}, & I_{1} & =\sum_{i, j, k, p, q, m=1}^{N} a_{i j k} a_{i j m}^{*} a_{p q m} a_{p q k}^{*}, \\
I_{2} & =\sum_{i, j, k, p, q, m=1}^{N} a_{i k j} a_{i m j}^{*} a_{p m q} a_{p k q}^{*}, & I_{3} & =\sum_{i, j, k, p, q, m=1}^{N} a_{k i j} a_{m i j}^{*} a_{m p q} a_{k p q}^{*} .
\end{aligned}
$$

A generalized concurrence is defined by [19],

$$
\begin{aligned}
C_{N}^{3} & =\sqrt{\frac{N}{3(N-1)}\left(3 I_{0}^{2}-I_{1}-I_{2}-I_{3}\right)} \\
& =\sqrt{\frac{N}{6(N-1)} \sum\left(\left|a_{i j k} a_{p q m}-a_{i j m} a_{p q k}\right|^{2}+\left|a_{i j k} a_{p q m}-a_{i q k} a_{p j m}\right|^{2}+\left|a_{i j k} a_{p q m}-a_{p j k} a_{i q m}\right|^{2}\right)} .
\end{aligned}
$$

First, we prove the following result:

Lemma 1. $C_{N}^{3}=0$ if and only if $|\Psi\rangle$ is separable.

Proof. It is clear that $C_{N}^{3}=0$ when $|\Psi\rangle$ is factorizable, i.e., when

$$
a_{i j k}=a_{i} b_{j} c_{k}, \text { for some } a_{i}, b_{j}, c_{k} \in \mathbb{C} .
$$

Conversely, because $|\Psi\rangle \neq 0$, there exists $p_{0}, q_{0}, m_{0}$ such that $a_{p_{0} q_{0} m_{0}} \neq 0$. Hence from $\mid a_{i j k} a_{p q m}-$ $a_{i j m} a_{p q k} \mid=0$ we have $a_{i j k}=a_{i j} b_{k}$, for some $a_{i j}, b_{k} \in \mathbb{C}$. Further we get $a_{i j k}=a_{i}^{\prime} b_{j}^{\prime} c_{k}^{\prime}, \quad a_{i}^{\prime}, b_{j}^{\prime}, c_{k}^{\prime} \in$ $\mathbb{C}$.

Let $\rho$ be a rank two state on $H \otimes H \otimes H$, with $\left|E_{1}\right\rangle,\left|E_{2}\right\rangle$ being its two orthonormal eigenvectors corresponding to the two nonzero eigenvalues:

$$
\rho=p\left|E_{1}\right\rangle\left\langle E_{1}|+q| E_{2}\right\rangle\left\langle E_{2}\right|,
$$

where $q=1-p \in(0,1)$. Generally

$$
\left|E_{s_{1}}\right\rangle=\sum_{i, j, k=1}^{N} a_{i j k}^{s_{1}} e_{i} \otimes e_{j} \otimes e_{k}, \quad a_{i j k}^{s_{1}} \in \mathbb{C},
$$


with normalization $\sum_{i, j, k=1}^{N} a_{i j k}^{s_{1}}\left(a_{i j k}^{s_{1}}\right)^{*}=1, s_{1}=1,2$.

Using Lemma 1, we have that $|\Psi\rangle=\sum_{i, j, k=1}^{N} a_{i j k} e_{i} \otimes e_{j} \otimes e_{k}$ is separable if and only if $C_{N}^{3}=0$, i.e.,

$$
a_{i j k} a_{p q m}=a_{i j m} a_{p q k}, \quad a_{i j k} a_{p q m}=a_{i q k} a_{p j m}, \quad a_{i j k} a_{p q m}=a_{p j k} a_{i q m}, \quad \forall i, j, k, p, q, m .
$$

We adopt the notation

$$
\begin{aligned}
& \alpha_{1}^{I}=a_{i j k}^{2} a_{p q m}^{2}-a_{i j m}^{2} a_{p q k}^{2}, \quad \alpha_{2}^{I}=a_{i j k}^{2} a_{p q m}^{2}-a_{i q k}^{2} a_{p j m}^{2}, \quad \alpha_{3}^{I}=a_{i j k}^{2} a_{p q m}^{2}-a_{p j k}^{2} a_{i q m}^{2}, \\
& \beta_{1}^{I}=a_{i j k}^{2} a_{p q m}^{1}+a_{i j k}^{1} a_{p q m}^{2}-a_{i j m}^{2} a_{p q k}^{1}-a_{i j m}^{1} a_{p q k}^{2}, \\
& \beta_{2}^{I}=a_{i j k}^{2} a_{p q m}^{1}+a_{i j k}^{1} a_{p q m}^{2}-a_{i q k}^{2} a_{p j m}^{1}-a_{i q k}^{1} a_{p j m}^{2}, \\
& \beta_{3}^{I}=a_{i j k}^{2} a_{p q m}^{1}+a_{i j k}^{1} a_{p q m}^{2}-a_{p j k}^{2} a_{i q m}^{1}-a_{p j k}^{1} a_{i q m}^{2}, \\
& \gamma_{1}^{I}=a_{i j k}^{1} a_{p q m}^{1}-a_{i j m}^{1} a_{p q k}^{1}, \quad \gamma_{2}^{I}=a_{i j k}^{1} a_{p q m}^{1}-a_{i q k}^{1} a_{p j m}^{1}, \quad \gamma_{3}^{I}=a_{i j k}^{1} a_{p q m}^{1}-a_{p j k}^{1} a_{i q m}^{1} .
\end{aligned}
$$

where $I=\{i, j, k, p, q, m\}, \forall i, j, k, p, q, m \in\{1,2, \ldots, N\}$.

A vector of the form $\left|E_{1}\right\rangle+\lambda\left|E_{2}\right\rangle, \lambda \in \mathbb{C}$, is separable if and only if $\lambda$ is a common root of the following equation set $E q_{s}^{I}$ :

$$
\alpha_{s}^{I} \lambda^{2}+\beta_{s}^{I} \lambda+\gamma_{s}^{I}=0
$$

where $s=1,2,3$, and $I=\{i, j, k, p, q, m\}, \forall i, j, k, p, q, m \in\{1,2, \ldots, N\}$.

Lemma 2. If $\left|E_{2}\right\rangle$ is not separable, then $\rho$ is separable if and only if (6) have two distinct roots.

Proof. Suppose that $\rho=\sum_{t=1}^{l} p_{t}^{\prime}\left|U_{t}\right\rangle\left\langle U_{t}\right|$, with $l$ some positive integer and $0<p_{t}^{\prime}<$ $1, \sum p_{t}^{\prime}=1,\left|U_{t}\right\rangle$ being separable, $\forall t$. We can write them as linear combinations of the two eigenvectors $\left|E_{1}\right\rangle$ and $\left|E_{2}\right\rangle$ which span the range of $\rho:\left|U_{t}\right\rangle=c_{1}^{t}\left|E_{1}\right\rangle+c_{2}^{t}\left|E_{2}\right\rangle$ (for some $c_{1}^{t}, c_{2}^{t} \in \mathbb{C}$ ). As $\left|U_{t}\right\rangle \neq 0, c_{1}^{t}, c_{2}^{t}$ can not be all 0 . Without losing generality, let $c_{1}^{t} \neq 0$. $\left|U_{t}\right\rangle$ is then of the form $\left|E_{1}\right\rangle+\lambda_{t}\left|E_{2}\right\rangle, \lambda_{t}=c_{2}^{t} / c_{1}^{t}$. From Lemma $1\left|U_{t}\right\rangle$ is separable if and only if the parameter $\lambda_{t}$ is a common root of the corresponding equation set (6), $\alpha_{s}^{I} \lambda_{t}^{2}+\beta_{s}^{I} \lambda_{t}+\gamma_{s}^{I}=0$.

Because $\left|E_{2}\right\rangle$ is not separable, not all $\lambda_{t}^{\prime} s$ can be equal, otherwise all the $U_{t}^{\prime} s$ would be constant multiples of a fixed vector, and $\rho$ would be rank 1 . On the other hand, as $\left|E_{2}\right\rangle$ is not separable, then $C_{N}^{3}$ is not zero. Hence there is some $I_{0}, s_{0}$ such that $\alpha_{s_{0}}^{I_{0}} \neq 0$, i.e., the relation $E q_{s}^{I}$ is indeed a quadratic equation. It must have exactly two roots, and so there are two values that are the only possible choices for the $\lambda_{t}^{\prime} s$. But in order that there is not only one possible choice, the above two roots must be different. And all the relations $E q_{s}^{I}$ have these two different roots.

Let $\mu_{1}, \mu_{2}$ be two distinct roots, which are common to all of the equations $E q_{s}^{I}$. Each vector $\left|U_{t}\right\rangle$ is either of the form $\left|E_{1}^{\prime}\right\rangle=\left(\left|E_{1}\right\rangle+\mu_{1}\left|E_{2}\right\rangle\right) / \sqrt{1+\left|\mu_{1}\right|^{2}}$, or of the form $\left|E_{2}^{\prime}\right\rangle=$ $\left(\left|E_{1}\right\rangle+\mu_{2}\left|E_{2}\right\rangle\right) / \sqrt{1+\left|\mu_{2}\right|^{2}}$. 
Therefore we can write $\rho$ as $\rho=p^{\prime}\left|E_{1}^{\prime}\right\rangle\left\langle E_{1}^{\prime}\left|+\left(1-p^{\prime}\right)\right| E_{2}^{\prime}\right\rangle\left\langle E_{2}^{\prime}\right|$ with $0<p^{\prime}<1$. Comparing the coefficients of $\left|E_{k}\right\rangle\left\langle E_{l}\right|, k, l=1,2$, with the ones in the expression (3), we get that the following two relations:

$$
\begin{gathered}
\frac{p^{\prime}}{1+\left|\mu_{1}\right|^{2}}+\frac{1-p^{\prime}}{1+\left|\mu_{2}\right|^{2}}=p \\
\frac{\mu_{1} p^{\prime}}{1+\left|\mu_{1}\right|^{2}}+\frac{\mu_{2}\left(1-p^{\prime}\right)}{1+\left|\mu_{2}\right|^{2}}=0
\end{gathered}
$$

Solving for $p$ and $p^{\prime}$ we get

$$
p=\left(1-\mu_{1} \mu_{2} \frac{\bar{z}}{z}\right)^{-1}, \quad p^{\prime}=\frac{\mu_{2}\left(1+\left|\mu_{1}\right|^{2}\right)}{z-\mu_{1} \mu_{2} \bar{z}}, \text { where } z=\mu_{2}-\mu_{1} .
$$

Conversely, let $\mu_{1}, \mu_{2}$ be two distinct roots, which are common to all of the equations $E q_{s}^{I}$. From above discussion we have $\rho=p^{\prime}\left|E_{1}^{\prime}\right\rangle\left\langle E_{1}^{\prime}\left|+\left(1-p^{\prime}\right)\right| E_{2}^{\prime}\right\rangle\left\langle E_{2}^{\prime}\right|$, i.e., $\rho$ is separable.

We first deal with the case where $a_{i j k}^{s_{1}}$ are all real. The following conclusion is easily verified.

Lemma 3. For a quadratic equation $a x^{2}+b x+c=0$ with $a, b, c \in \mathbb{R}, a \neq 0$, and roots $\alpha, \beta$ with $\alpha \neq \beta, \gamma=(\bar{\alpha}-\bar{\beta}) /(\alpha-\beta)$ is either 1 or -1 .

Theorem 1. If all $a_{i j k}^{s_{1}}$ are real, $\rho$ is separable if and only if one of the following quantities $\left(\triangle_{1}\right.$ or $\triangle_{2}$ ) is zero:

$$
\begin{gathered}
\triangle_{1}=\sum\left|\gamma_{s}^{I}-\left(1-p^{-1}\right) \alpha_{s}^{I}\right|^{2}+\sum\left|\beta_{s}^{I} \alpha_{s^{\prime}}^{I^{\prime}}-\alpha_{s}^{I} \beta_{s^{\prime}}^{I^{\prime}}\right|^{2}, \\
\triangle_{2}=\sum\left|\gamma_{s}^{I}+\left(1-p^{-1}\right) \alpha_{s}^{I}\right|^{2}+\sum\left|\beta_{s}^{I}\right|^{2},
\end{gathered}
$$

or, equivalently, one of the following two sets of relations (12) or (13) hold:

$$
\begin{gathered}
\gamma_{s}^{I}=\left(1-p^{-1}\right) \alpha_{s}^{I}, \quad \beta_{s}^{I} \alpha_{s^{\prime}}^{I^{\prime}}=\alpha_{s}^{I} \beta_{s^{\prime}}^{I^{\prime}} \\
\gamma_{s}^{I}=-\left(1-p^{-1}\right) \alpha_{s}^{I}, \quad \beta_{s}^{I}=0
\end{gathered}
$$

where $s, s^{\prime}=1,2,3$, and $I, I^{\prime}=\{i, j, k, p, q, m\}, \forall i, j, k, p, q, m \in\{1,2, \ldots, N\}$.

Proof. We prove the necessity part of the theorem in two cases:

Case 1. a). $\left|E_{2}\right\rangle$ is not separable. We get that (6) have two distinct roots from Lemma 2. These two roots are the solutions to all the relations $E q_{s}^{I}$. Consider for any $s=1,2,3, I=$ $\{i, j, k, p, q, m\}, \forall i, j, k, p, q, m \in\{1,2, \ldots, N\}$,

1) if $\alpha_{s}^{I} \neq 0$, the corresponding relation (6) is not an identity. All the quadratic equations in the set $E q_{s}^{I}$ have the same two distinct roots. From the standard theory of quadratic equations, we have

$$
\begin{aligned}
& \beta_{s}^{I} \alpha_{s_{0}}^{I_{0}}=\beta_{s_{0}}^{I_{0}} \alpha_{s}^{I}, \\
& \gamma_{s}^{I} \alpha_{s_{0}}^{I_{0}}=\gamma_{s_{0}}^{I_{0}} \alpha_{s}^{I} .
\end{aligned}
$$


2) if $\alpha_{s}^{I}=0$, then the equations $E q_{s}^{I}$ become identities, i.e., $\beta_{s}^{I}$ and $\gamma_{s}^{I}$ must be 0 too, because otherwise at least one of the relations $E q_{s}^{I}$ would be a linear equation, and there would be no two distinct roots. Thus in this case (12), (13) also hold.

b). Because all $a_{i j k}^{s_{1}}$ are real number, $\mu_{1}$ and $\mu_{2}$ are roots of a quadratic equation with real coefficients. From Lemma $3, \mu_{1} \mu_{2}=1-p^{-1}$ or $-\left(1-p^{-1}\right)$. Since $\mu_{1} \mu_{2}$ is real, the solution for $p^{\prime}$ in (9) implies that $\mu_{2} /\left(\mu_{2}-\mu_{1}\right)$ is real, which is possible if and only if either the roots are both real or the roots are both purely imaginary. In the first case, let $\mu_{2}>\mu_{1}$, we have $\mu_{1} \mu_{2}=1-p^{-1}$. From (9), we get the condition that $p^{\prime} \in[0,1]$, which is equivalent to $\mu_{2}>0, \mu_{1}<0$. In the second case, we have $\mu_{1} \mu_{2}=-\left(1-p^{-1}\right)$. The condition for having purely imaginary roots of quadratic equations gives that $\beta_{s}^{I}=0, \forall I, \forall s$.

c). Finally, we observe that $\mu_{1} \mu_{2}$ is nothing but the ratio $\gamma_{s_{0}}^{I_{0}} / \alpha_{s_{0}}^{I_{0}}$, which is either $1-p^{-1}$ or $-\left(1-p^{-1}\right)$. Therefore we conclude that either $\gamma_{s}^{I}=\left(1-p^{-1}\right) \alpha_{s}^{I}$ or $\gamma_{s}^{I}=-\left(1-p^{-1}\right) \alpha_{s}^{I}$ for any $I$ and $s=1,2,3$. Relation (10) is verified.

Case 2. $\left|E_{2}\right\rangle$ is separable. In this case from (4), we have $\alpha_{s}^{I}=0, \forall I, \forall s$. Since not all of the $\left|U_{t}\right\rangle$ can be multiples of $\left|E_{2}\right\rangle$, we must have at least one choice of $\lambda$ such that $\left|E_{1}\right\rangle+\lambda\left|E_{2}\right\rangle$ is separable. This must be a common root to all equations $E q_{s}^{I}$ as before. All these equations are now linear ones. When all $\beta_{s}^{I}=\gamma_{s}^{I}=0$, it is easy to see that $\left|E_{1}\right\rangle$ is separable. Excluding this case, we see that there is only one possible choice of $\lambda$. Then $\rho$ can be expressed as

$$
\rho=p^{\prime \prime}\left|E_{2}\right\rangle\left\langle E_{2}\right|+\left(1-p^{\prime \prime}\right) \frac{\left|E_{1}+\lambda E_{2}\right\rangle\left\langle E_{1}+\lambda E_{2}\right|}{1+|\lambda|^{2}} .
$$

That is $p^{\prime \prime}=1$, which is a contradiction. Thus, if $\left|E_{2}\right\rangle$ is separable, $\left|E_{1}\right\rangle$ must be separable too. It is clear that in this case (10) and (11) hold.

Now we prove the sufficiency part for the theorem. If (10) or (11) holds, it is clear the equations $E q_{s}^{I}$ have common roots. If $\left|E_{2}\right\rangle$ is not separable, then not all of these equations are identities. And there are at most two common roots. If (10) holds, the product of the two roots must be $1-p^{-1}<0$, so that the two roots are real and unequal. If (11) holds, the two roots must be purely imaginary. So in these two cases, we get that $\rho$ is separable in terms of Lemma 2. If $\left|E_{2}\right\rangle$ is separable, from (10) or (11) we know $\left|E_{1}\right\rangle$ is separable too and $\rho$ is separable.

Generalizing the results in Theorem 1, we have, for the complex $a_{i j k}^{s_{1}}$,

Theorem 2. $\rho$ is separable if and only if there is $\theta \in \mathbb{R}$ such that

$$
\gamma_{s}^{I}=e^{i \theta}\left(1-p^{-1}\right) \alpha_{s}^{I}, \quad \beta_{s}^{I} \alpha_{s^{\prime}}^{I^{\prime}}=\alpha_{s}^{I} \beta_{s^{\prime}}^{I^{\prime}}
$$

where $s, s^{\prime}=1,2,3$, and $I, I^{\prime}=\{i, j, k, p, q, m\}, \forall i, j, k, p, q, m \in\{1,2, \ldots, N\}$, and

$$
\frac{\mu_{2}\left(1+\left|\mu_{1}\right|^{2}\right)}{z-\mu_{1} \mu_{2} \bar{z}} \in[0,1]
$$

where $z=e^{i \theta} \bar{z}, z=\mu_{2}-\mu_{1} \neq 0, \mu_{1}$ and $\mu_{2}$ are the roots of the equation $\alpha_{s}^{I} \lambda^{2}+\beta_{s}^{I} \lambda+\gamma_{s}^{I}=0$ for some $I$ and $s$ such that $\alpha_{s}^{I} \neq 0$. 
Proof. The proof of necessity is similar to the proof of the corresponding part in Theorem 1. One only needs to note that since $z / \bar{z}$ is of modulus 1 , a phase factor $e^{i \theta}$ appears in this case.

Now if (14) holds, it is clear that the equations $E q_{s}^{I}$ have common roots. If $\left|E_{2}\right\rangle$ is not separable, then some of the $\alpha_{s}^{I}$ are nonzero. The corresponding equations $E q_{s}^{I}$ have exactly two roots which are different by condition (15). Therefore $\rho$ is separable from Lemma 2. If $\left|E_{2}\right\rangle$ is separable, by (14) we know that all $\gamma_{s}^{I}$ are 0 . Hence both $\left|E_{2}\right\rangle$ and $\left|E_{1}\right\rangle$ are separable, and so is $\rho$.

Corollary. Let $\left|E_{2}\right\rangle$ be the maximally entangled vector given by $\left|E_{2}\right\rangle=(1 / \sqrt{N}) \sum_{i=1}^{N} e_{i} \otimes$ $e_{i} \otimes e_{i}$. For any vector $\left|E_{1}\right\rangle$ which is orthogonal to $\left|E_{2}\right\rangle, \rho=p\left|E_{1}\right\rangle\left\langle E_{1}|+(1-p)| E_{2}\right\rangle\left\langle E_{2}\right|$ is not separable for $0<p<1 / 2$.

Proof. Let

$$
C_{(1)}=\sqrt{\frac{N}{6(N-1)} \sum_{I, s}\left|\gamma_{s}^{I}\right|^{2}} \text { and } C_{(2)}=\sqrt{\frac{N}{6(N-1)} \sum_{I, s}\left|\alpha_{s}^{I}\right|^{2}}
$$

be the generalized concurrences associated with the states $\left|E_{1}\right\rangle$ and $\left|E_{2}\right\rangle$, respectively, where $s=1,2,3, I=\{i, j, k, p, q, m\}, \forall i, j, k, p, q, m \in\{1,2, \ldots, N\}$.

Suppose $\rho$ is separable. From the necessary condition for separability, $\gamma_{s}^{I}=e^{i \theta}\left(1-p^{-1}\right) \alpha_{s}^{I}$, we get $C_{(1)}=\frac{1-p}{p} C_{(2)}$. As $\left|E_{2}\right\rangle$ is maximally entangled, $C_{(2)} \neq 0$, and $C_{(1)} / C_{(2)}=\frac{1-p}{p} \leq 1$, so we have $p \geq 1 / 2$, which is a contradiction.

The above approach can be extended to the case of multiquantum systems. We consider now the separability of $\left|\Psi_{M}\right\rangle$ on $M N$-dimensional quantum systems, where

$$
\left|\Psi_{M}\right\rangle=\sum_{i_{1}, i_{2}, \ldots, i_{M}=1}^{N} a_{i_{1} i_{2} \ldots i_{M}} e_{i_{1}} \otimes e_{i_{2}} \otimes \ldots \otimes e_{i_{M}}, \quad a_{i_{1} i_{2} \ldots i_{M}} \in \mathbb{C}
$$

with $\sum a_{i_{1} i_{2} \ldots i_{M}} a_{i_{1} i_{2} \ldots i_{M}}^{*}=1$. We have a quadratic $I_{0}=\sum a_{i_{1} i_{2} \ldots i_{M}} a_{i_{1} i_{2} \ldots i_{M}}^{*}$ and $d=2^{M-1}-1$ biquadratic invariants:

$$
I_{T S}=\sum a_{T S} a_{T S^{\prime}}^{*} a_{T^{\prime} S^{\prime}} a_{T^{\prime} S}^{*}
$$

where $T$ and $T^{\prime}$ are all possible nontrivial subset of $I=\left\{i_{1}, i_{2}, \ldots, i_{M}\right\}, I^{\prime}=\left\{\tilde{i_{1}}, \tilde{i_{2}}, \ldots, \tilde{i_{M}}\right\}$, respectively, $\forall i_{k}, \tilde{i_{k}}=1,2, \cdots, N, k=1,2, \ldots, M$ (i.e., $T \neq \phi$ and $T \neq I$ ), $S=I \backslash T, S^{\prime}=I^{\prime} \backslash T^{\prime}$.

$T$ and $T^{\prime}$ are subindices of $a$, associated with the same position. A generalized concurrence can be defined by

$$
C_{N}^{M}=\sqrt{\frac{N}{d(N-1)}\left(d I_{0}^{2}-I_{1}-I_{2}-\cdots-I_{d}\right)}=\sqrt{\frac{N}{4 d(N-1)} \sum_{p}\left|a_{T S} a_{T^{\prime} S^{\prime}}-a_{T S^{\prime}} a_{T^{\prime} S}\right|^{2}}
$$

where $\sum_{p}$ stands for the summation over all possible combination of the indices of $T$ and $S$. Similar to Lemma 1, one can prove: 
Lemma 4. $C_{N}^{M}=0$ if and only if $\left|\Psi_{M}\right\rangle$ is separable.

Let $\rho$ be a rank two state on $H \otimes H \otimes \cdots \otimes H$, with $\left|E_{1}\right\rangle,\left|E_{2}\right\rangle$ being its two orthonormal eigenvectors corresponding to the two nonzero eigenvalues:

$$
\rho=p\left|E_{1}\right\rangle\left\langle E_{1}|+q| E_{2}\right\rangle\left\langle E_{2}\right|
$$

where $q=1-p \in(0,1)$. Generally

$$
\left|E_{s_{1}}\right\rangle=\sum_{i_{1} i_{2} \ldots i_{M}=1}^{N} a_{i_{1} i_{2} \ldots i_{M}}^{s_{1}} e_{i_{1}} \otimes e_{i_{2}} \otimes \ldots \otimes e_{i_{M}}, \quad a_{i_{1} i_{2} \ldots i_{M}}^{s_{1}} \in \mathbb{C}
$$

with normalization $\sum a_{i_{1} i_{2} \ldots i_{M}}^{s_{1}}\left(a_{i_{1} i_{2} \ldots i_{M}}^{s_{1}}\right)^{*}=1, s_{1}=1,2$.

Using Lemma 4, we have $\left|\Psi_{M}\right\rangle$ is separable if and only if

$$
a_{T S} a_{T^{\prime} S^{\prime}}=a_{T S^{\prime}} a_{T^{\prime} S}
$$

where $T$ and $T^{\prime}$ are all possible nontrivial subset of $I=\left\{i_{1}, i_{2}, \ldots, i_{M}\right\}, I^{\prime}=\left\{\tilde{i_{1}}, \tilde{i_{2}}, \ldots, \tilde{i_{M}}\right\}$, respectively, $\forall i_{k}, \tilde{i_{k}}=1,2, \ldots, N, k=1,2, \ldots, M$ (i.e., $T \neq \phi$ and $T \neq I$ ), $S=I \backslash T, S^{\prime}=I^{\prime} \backslash T^{\prime}$.

With the notations:

$$
\begin{aligned}
& \alpha_{T S}^{T^{\prime} S^{\prime}}=a_{T S}^{2} a_{T^{\prime} S^{\prime}}^{2}-a_{T S^{\prime}}^{2} a_{T^{\prime} S}^{2}, \quad \gamma_{T S}^{T^{\prime} S^{\prime}}=a_{T S}^{1} a_{T^{\prime} S^{\prime}}^{1}-a_{T S^{\prime}}^{1} a_{T^{\prime} S}^{1}, \\
& \beta_{T S}^{T^{\prime} S^{\prime}}=a_{T S}^{2} a_{T^{\prime} S^{\prime}}^{1}+a_{T S}^{1} a_{T^{\prime} S^{\prime}}^{2}-a_{T S^{\prime}}^{2} a_{T^{\prime} S}^{1}-a_{T S^{\prime}}^{1} a_{T^{\prime} S}^{2},
\end{aligned}
$$

we have that a vector of the form $\left|E_{1}\right\rangle+\lambda\left|E_{2}\right\rangle, \lambda \in \mathbb{C}$, is separable if and only if $\lambda$ is a common root of the following equation set:

$$
E q_{T S}^{T^{\prime} S^{\prime}}: \alpha_{T S}^{T^{\prime} S^{\prime}} \lambda^{2}+\beta_{T S}^{T^{\prime} S^{\prime}} \lambda+\gamma_{T S}^{T^{\prime} S^{\prime}}=0
$$

Similar to the case $M=3$, one has:

Lemma 5. $\rho$ is separable if and only if (20) have two distinct roots.

From Lemma 4 and Lemma 5 it is straightforward to prove the following conclusion:

Theorem 3. If all $a_{i_{1} i_{2} \ldots i_{M}}^{s_{1}}$ are real, $\rho$ is separable if and only if one of the following quantities $\left(\triangle_{1}\right.$ or $\left.\triangle_{2}\right)$ is zero:

$$
\begin{gathered}
\triangle_{1}=\sum\left|\gamma_{T S}^{T^{\prime} S^{\prime}}-\left(1-p^{-1}\right) \alpha_{T S}^{T^{\prime} S^{\prime}}\right|^{2}+\sum\left|\beta_{T S}^{T^{\prime} S^{\prime}} \alpha_{T_{1} S_{1}}^{T_{1}^{\prime} S_{1}^{\prime}}-\alpha_{T S}^{T^{\prime} S^{\prime}} \beta_{T_{1} S_{1}}^{T_{1}^{\prime} S_{1}^{\prime}}\right|^{2}, \\
\triangle_{2}=\sum\left|\gamma_{T S}^{T^{\prime} S^{\prime}}+\left(1-p^{-1}\right) \alpha_{T S}^{T^{\prime} S^{\prime}}\right|^{2}+\sum\left|\beta_{T S}^{T^{\prime} S^{\prime}}\right|^{2}
\end{gathered}
$$

or, equivalently, one of the following two sets of relations (23) or (24) hold:

$$
\begin{gathered}
\gamma_{T S}^{T^{\prime} S^{\prime}}=\left(1-p^{-1}\right) \alpha_{T S}^{T^{\prime} S^{\prime}}, \quad \beta_{T S}^{T^{\prime} S^{\prime}} \alpha_{T_{1} S_{1}}^{T_{1}^{\prime} S_{1}^{\prime}}=\alpha_{T S}^{T^{\prime} S^{\prime}} \beta_{T_{1} S_{1}}^{T_{1}^{\prime} S_{1}^{\prime}} \\
\gamma_{T S}^{T^{\prime} S^{\prime}}=-\left(1-p^{-1}\right) \alpha_{T S}^{T^{\prime} S^{\prime}}, \quad \beta_{T S}^{T^{\prime} S^{\prime}}=0
\end{gathered}
$$


where $T$ and $T^{\prime}$ are all possible nontrivial subset of $I=\left\{i_{1}, i_{2}, \ldots, i_{M}\right\}, I^{\prime}=\left\{\tilde{i_{1}}, \tilde{i_{2}}, \ldots, \tilde{i_{M}}\right\}$, respectively, $\forall i_{k}, \tilde{i_{k}}=1,2, \ldots, N, k=1,2, \ldots, M$ (i.e., $T \neq \phi$ and $T \neq I$ ), $S=I \backslash T, S^{\prime}=$ $I^{\prime} \backslash T^{\prime}, T_{1}$ and $T_{1}^{\prime}$ are all possible nontrivial subset of $J=\left\{j_{1}, j_{2}, \ldots, j_{M}\right\}, J^{\prime}=\left\{\tilde{j_{1}}, \tilde{j_{2}}, \ldots, \tilde{j_{M}}\right\}$, respectively, $\forall j_{k}, \tilde{j_{k}}=1,2, \ldots, N, k=1,2, \ldots, M$ (i.e., $T_{1} \neq \phi$ and $T_{1} \neq J$ ), $S_{1}=J \backslash T_{1}, S_{1}^{\prime}=$ $J_{1}^{\prime} \backslash T_{1}^{\prime}$.

Extending Theorem 3 to general complex coefficients $a_{i_{1} i_{2} \ldots i_{M}}^{s_{1}}$, we have

Theorem 4. $\rho$ is separable if and only if there is $\theta \in \mathbb{R}$ such that

$$
\begin{gathered}
\gamma_{T S}^{T^{\prime} S^{\prime}}=e^{i \theta}\left(1-p^{-1}\right) \alpha_{T S}^{T^{\prime} S^{\prime}}, \beta_{T S}^{T^{\prime} S^{\prime}} \alpha_{T_{1} S_{1}}^{T_{1}^{\prime} S_{1}^{\prime}}=\alpha_{T S}^{T^{\prime} S^{\prime}} \beta_{T_{1} S_{1}}^{T_{1}^{\prime} S_{1}^{\prime}} \\
\frac{\mu_{2}\left(1+\left|\mu_{1}\right|^{2}\right)}{z-\mu_{1} \mu_{2} \bar{z}} \in[0,1] .
\end{gathered}
$$

where $T, T^{\prime}, S, S^{\prime}, T_{1}, T_{1}^{\prime}, S_{1}, S_{1}^{\prime}$ are defined as in Theorem $3, z=e^{i \theta} \bar{z}, z=\mu_{2}-\mu_{1} \neq 0, \mu_{1}$ and $\mu_{2}$ are the roots of the equation $\alpha_{T S}^{T^{\prime} S^{\prime}} \lambda^{2}+\beta_{T S}^{T^{\prime} S^{\prime}} \lambda+\gamma_{T S}^{T^{\prime} S^{\prime}}=0$ for some $T, S, T^{\prime}, S^{\prime}$ such that $\alpha_{T S}^{T^{\prime} S^{\prime}} \neq 0$.

For a given rank two density matrix on $H \otimes H \otimes \cdots \otimes H$, to find its separability one only needs to calculate the two eigenvectors $\left|E_{1}\right\rangle,\left|E_{2}\right\rangle$ corresponding to the two nonzero eigenvalues and check if formula (25) is satisfied or not.

Corollary. Let $\left|E_{2}\right\rangle$ be the maximally entangled vector given by $\left|E_{2}\right\rangle=(1 / \sqrt{N}) \sum_{i=1}^{N} e_{i} \otimes$ $e_{i} \otimes \cdots \otimes e_{i}$. For any vector $\left|E_{1}\right\rangle$ which is orthogonal to $\left|E_{2}\right\rangle, \rho=p\left|E_{1}\right\rangle\left\langle E_{1}|+(1-p)| E_{2}\right\rangle\left\langle E_{2}\right|$ is not separable for $0<p<1 / 2$.

We have studied the sufficient and necessary conditions for separability of rank two mixed states in higher-dimensional quantum systems on $H \otimes H \otimes \ldots \otimes H$. The separability condition for these kind of mixed states in arbitrary dimensions is explicitly given. A nonseparability inequality is also given for the case where one of the eigenvectors corresponding to nonzero eigenvalues of a density matrix is maximally entangled. The results can be generalized to the case of rank two mixed states on $H_{1} \otimes H_{2} \otimes \ldots \otimes H_{M}$, where $H_{i}, i=1, \ldots, M$, may have different dimensions.

Acknowledgement The work is supported by the NSF of China (No.19975061) and the National Key Project for Basic Research of China (G1998030601).

\section{References}

[1] A. Ekert, Phys. Rev. Lett. 67, 661 (1991).

[2] C. H. Bennett and S. J. Wiesner, Phys. Rev. Lett. 69, 2881 (1992).

[3] C. Bennett, G. Brassard, C. Crepeau, R. Jozsa, A. Peres and W. K. Wootters, Phys. Rev. Lett. 70, 1895 (1993). 
[4] A. Peres, "Quantum Theory: Concepts and Methods", Kluwer Academic Publishers (1995).

[5] see M. Horodecki, P. Horodecki and R. Horodecki in "Quantum Information - Basic Concepts and Experiments", Eds. G. Alber and M. Weiner, (Springer, Berlin, 2000).

M. Lewenstein, D. Bruß, J. I. Cirac, B. Kraus, M. Kuś, J. Samsonowicz, A. Sanpera, and R. Tarrach, J. Mod. Phys. 47, 2481 (2000).

[6] R. Werner, Phys. Rev. A40, 4277 (1989).

[7] A. Peres Phys. Rev. Lett. 77, 1413 (1996).

[8] M. Horodecki, P. Horodecki, and R. Horodecki, Phys. Lett. A 223, 8 (1996).

[9] P. Horodecki Phys. Lett. A 232, 333 (1997).

[10] D. Bouwmeester, A. Ekert, and A. Zeilinger (Eds.), "The Physics of Quantum Information", (Springer, Heidelberg, 2000), chapter 6 and references therein.

[11] W. Dür, G. Vidal and J.I. Cirac, Phys. Rev. A 62, 062314(2000).

V. Coffman, J. Kundu ans W. Wootters, Phys. Rev. A 61, 052306(2000).

W. Wootters, Phys. Rev. A 63, 052302(2001).

[12] W. Tittel, H. Zbinden and N. Gisin, Quantum secret sharing using pseudo-GHZ states, quant-ph/9912035.

[13] A. Zeilinger, M. A. Horne, H. Weinfurter, and M. Żukowski, Phys. Rev. Lett. 78, 3031 (1997).

D. Bouwmeester, J-W. Pan, M. Daniell, H. Weinfurter, and A. Zeilinger, Phys. Rev. Lett. 82, 1345 (1999).

A. Sørensen and K. Mølmer, Phys. Rev. Lett. 82, 1971(1999).

C. A. Sackett, D. Kielpinski, B. E. King, C. Langer, V. Meyer, C. J. Myatt, M. Rowe, Q.

A. Turchette, W. M. Itano, D. J. Wineland, and C. Monroe, Nature 404, 256 (2000).

D. Kielpinski, A. Ben-Kish, J. Britton, V. Meyer, M.A. Rowe, C. A. Sackett, W. M. Itano, C. Monroe, and D. J. Wineland, Recent Results in Trapped-Ion Quantum Computing, quantph/0102086.

[14] H.J. Briegel and R. Raussendorf, Phys. Rev. Lett. 86, 910(2001).

[15] J. Eisert and H.J. Briegel, Phys. Rev. A 64, 022306(2001).

[16] S. Karnas and M. Lewenstein, Separability and entanglement in $\mathcal{C}^{2} \otimes \mathcal{C}^{2} \otimes \mathcal{C}^{N}$ composite quantum systems, quant-ph/0102115.

[17] Albeverio S., Fei S. M. and Goswami, D., Separability of rank two quantum states, Phys. Lett. A 286(2001)91-96. 
see also P. Horodecki, J.A. Smolin, B.M. Terhal and A.V. Thapliyal, Rank Two Bipartite Bound Entangled States Do Not Exist, quant-ph/9910122.

P. Horodecki, M. Lewenstein, G. Vidal and I. Cirac, Phys. Rev. A 62, 032310 (2000).

[18] N. Linden and P. Popescu, Fortsch. Phys. 46, 567 (1998).

[19] Albeverio S., Fei S. M., J. Opt. B: Quantum Semiclass. Opt. 3, 223(2001). 